\title{
Invertebrados exóticos nuevos o poco conocidos en la Ciudad de Buenos Aires
}

\author{
New or poorly known exotic invertebrates from \\ Buenos Aires City
}

Federico L. Agnolin ${ }^{1,2,3^{*}}$; Agustín Agnolin'; Elián L. Guerrero ${ }^{5}$

1 Laboratorio de Anatomía Comparada y Evolución de los Vertebrados, Museo Argentino de Ciencias Naturales "Bernardino Rivadavia". Av. Ángel Gallardo 470, (C1405DJR) Ciudad Autónoma de Buenos Aires, Argentina. CONICET. fedeagnolin@yahoo.com.ar.

2 Área Paleontología, Departamento de Ciencias Naturales y Antropología, Fundación de Historia Natural "Félix de Azara", Universidad Maimónides. Hidalgo 775 Piso 7, (C1405BDB) Ciudad Autónoma de Buenos Aires, Argentina.

3 Instituto Nacional de Antropología y Pensamiento Latinoamericano, 3 de Febrero 1378, (C1426BJN) Ciudad Autónoma de Buenos Aires, Argentina. CONICET. agusagnolin@yahoo.com.ar.

4 División Plantas Vasculares, Facultad de Ciencias Naturales y Museo, Universidad Nacional de La Plata, Paseo del Bosque s/n, (1900) La Plata, Buenos Aires, Argentina. CONICET.

eguerrero@fcnym.unlp.edu.ar

\section{RESUMEN}

Las especies invasoras pueden causar diversos problemas a nivel socioeconómico y para la conservación de la biodiversidad. La detección temprana de las especies naturalizadas puede ser de vital importancia para el monitoreo y la planificación de estrategias de contención del proceso de invasión. La fauna de planarias terrestres (Tricladida, Geoplanoidea), babosas terrestres (Mollusca, Limacidae), diplópodos (Myriapoda, Diplopoda) y sínfilos (Myriapoda, Symphyla) de la Ciudad Autónoma de Buenos Aires se encuentra poco estudiada, y la adición de especies exóticas transportadas por las actividades humanas complejiza la situación de poco conocimiento. En este trabajo se dan a conocer nuevos registros de especies de estos grupos que se han naturalizado en el ámbito urbano de la ciudad y sus alrededores (Bipalium kewense, Austroplana sanguinea, Ambigolimax valentianus, Brachyiulus pusillus, Oxidus gracilis y Scutigerella immaculata). Se evaluó el potencial como invasor de cada especie en base a lo registrado en otros países. Hasta el día de la fecha no existen registros publicados de planarias terrestres, sínfilos, o diplópodos para esta área, por lo cual el

\footnotetext{
Ref. bibliográfica: Agnolin, F. L.; Agnolin, A.; Guerrero, E. L. 2019. "Invertebrados exóticos nuevos o poco conocidos en la Ciudad de Buenos Aires". Acta zoológica lilloana 63 (2): 48-67. Fundación Miguel Lillo, Tucumán, Argentina. D.O.I.: https://doi.org/10.30550/j.azl/2019.63.2/4

> Recibido: 16/05/19 - Aceptado: 16/09/19

> URL de la revista: http://actazoologica.lillo.org.ar

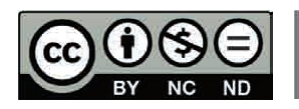

open $\hat{O}_{\text {ACCESS }}$

Algunos derechos reservados. Esta obra está bajo una Licencia Creative Commons Atribución - No Comercial - Sin Obra Derivada 4.0 Internacional.
} 
presente trabajo constituye la primera contribución sobre estos grupos. Las especies mencionadas perjudican a las actividades agropecuarias y horticulturales de diversas formas. Una tarea a futuro deberá ser la cuantificación de los daños que causan para establecer su importancia y el costo/beneficio de su control o manejo.

Palabras clave - Ciudad Autónoma de Buenos Aires, Geoplanoidea, Limacidae, Diplopoda, Symphyla.

\begin{abstract}
Exotic invertebrates in the city of Buenos Aires. First steps for the monitoring of potential biological invasions. Invasive species can cause several problems at the socio-economic level and for the biodiversity conservation. The early detection of naturalized species can be of vital importance for the planning of containment strategies of the invasion process. The flat worms (Tricladida, Geoplanoidea), land slugs (Mollusca, Limacidae), millipedes (Myriapoda, Diplopoda) and symphillids (Myriapoda, Symphyla) of the Ciudad Autónoma de Buenos Aires it is little studied, and the addition of exotic species transported by human activities complicates the situation of little knowledge. In this work, we present new records of species of these groups that have become naturalized in the urban area of the city and its surroundings (Bipalium kewense, Austroplana sanguinea, Ambigolimax valentianus, Brachyiulus pusillus, Oxidus gracilis y Scutigerella immaculata). The invasive potential of each species was evaluated based on what was registered in other countries. Until the date there are no published records of terrestrial flat worms, symphillids, or millipedes for this area, for which the present work constitutes the first contribution on these groups. The aforementioned species damage agricultural and horticultural activities in different ways. A future task should be the quantification of the damage caused to establish its importance and the cost / benefit of its control or management.
\end{abstract}

Keywords - Ciudad Autónoma de Buenos Aires, Geoplanoidea, Limacidae, Diplopoda, Symphyla.

\title{
INTRODUCCIÓN
}

Cuando una especie exótica produce daños en los ecosistemas nativos o en las actividades productivas de los humanos, o es esperable que los cause, se la considera invasora (Beck et al., 2008). El estudio del proceso de naturalización de especies exóticas es apremiante, debido a que éstas pueden inducir el desplazamiento de especies nativas, daños en cultivos, y otros daños ecológicos y económicos (Simberloff, 2005; Richardson y Ricciardi, 2013). La detección temprana de las especies exóticas que pueden devenir en invasoras y su estudio puede ayudar a disminuir los procesos negativos y evaluar la forma de obtener beneficios a partir de ellas (Hulme, 2009; Goodenough, 2010). Esta predicción anticipada es necesaria pero muy dificultosa en algunos casos, ya que al detectarse una especie exótica hay pocos —o nulos- in- 
dicadores de cuánto daño provocará (o cuan detectable será el daño a corto plazo) en los ecosistemas; solo se sabe si una especie es invasora en una región cuando ya causa problemas en ella. Sin embargo, cuando determinada especie ya ha causado problemas en otras regiones del globo, es prioritario alertar a los productores y ambientalistas para prevenir su expansión.

En el espacio urbano de la Ciudad Autónoma de Buenos Aires y alrededores, los jardines, viveros y huertos juegan un rol vital en la naturalización de especies de plantas exóticas (Hurrell, Delucchi, Keller, Stampella y Guerrero, 2012). Es posible que estos ámbitos sean también el centro de dispersión de invertebrados exóticos. Por ejemplo, la araña Badumna longinqua (Koch, 1857) (Desidae) se ha establecido primariamente en parques y plantaciones de Eucalyptus spp. (Pompozzi, Peralta, Simó, 2013). En la provincia de Buenos Aires se ha propuesto que la introducción de especies vegetales y animales desde la colonización europea pudo haber provocado el desplazamiento o la extinción de especies nativas de la región provocando un marcado descenso de los valores de riqueza con respecto a los valores esperables para la situación geográfico-climática del área (Rapoport, 1996).

La Ciudad Autónoma de Buenos Aires constituye el ejido urbano de mayor tamaño en Argentina. Con 203 kilómetros cuadrados y una población que ronda los 3 millones de habitantes, constituye una de las ciudades más densamente pobladas de la región. A pesar de su importancia y accesibilidad, la flora y fauna locales aún distan de ser bien conocidas. Publicaciones relacionadas a fauna local son escasas, y poco sabemos acerca de la fauna y flora adventicia que puede encontrarse en plazas y jardines urbanos. La mayor parte de los relevamientos se restringen especialmente a árboles, aves y mariposas (Barreiro, 2011; Landolfi, 2014; Narosky y Henschke, 2005; Núñez Bustos, 2008; 2010). Los estudios están especialmente sesgados hacia el área protegida de la Reserva Ecológica Costanera Sur, donde se tiene una variada información sobre la estructura vegetal (Faggi y Cagnoni, 1987), aves (Filipello y López de Casenave, 1993; López de Casenave y Filipello, 1995), e incluso artrópodos (Fontanarrosa, Torres y Michat, 2004; Mulieri, Torretta, Schnackand, Mariluis, 2006; Nuñez Bustos, 2008; Carpintero, De Biase, Konopko, 2014; Guerrero, 2014; Zapata y Grismado, 2015). Los relevamientos de Araneae realizados en la última década ilustran adecuadamente la necesidad de incrementar los estudios en el área: en la capital del país quedan numerosas especies por describir (Zapata y Grismado, 2015) y algunas especies exóticas por ser descubiertas (Pompozzi, et al., 2013).

La fauna de planarias terrestres (Tricladida, Geoplanoidea), babosas terrestres (Mollusca, Limacidae), diplópodos (Myriapoda, Diplopoda) y sínfilos (Myriapoda, Symphyla) de la Ciudad Autónoma de Buenos Aires se encuentra poco estudiada, y la adición de especies exóticas transportadas por las actividades humanas complejiza la situación de escaso conocimiento. La finalidad de la presente contribución es reportar el establecimiento de especies no nativas de invertebrados de los grupos taxonómicos mencionados y evaluar el potencial como invasor de cada una de ellas. Hasta el día de la fecha no existen registros publicados de planarias terrestres, sínfilos, o diplópodos para la Ciudad Autónoma de Buenos Aires, por lo cual el presente trabajo constituye la primera contribución sobre estos grupos para la localidad. 


\title{
MATERIALES Y MÉTODOS
}

Se recolectaron los Tricladida, Mollusca y Myriapoda hallados en jardines de la Ciudad Autónoma de Buenos Aires y se determinaron mediante bibliografía específica para cada grupo (la cual es detallada en la sección del texto que corresponde a cada taxón). El material de referencia proviene mayormente del patio de una vivienda de una zona densamente poblada, ubicada en el barrio de Liniers, a la altura de la avenida Rivadavia al 10700 . Este patio posee plantas decorativas tanto nativas como exóticas, con piedras y ladrillos que brindan refugio a los invertebrados. Adicionalmente se recolectaron especímenes de algunas especies en la Ciudad de La Plata, ubicada a 50 kilómetros de allí. En esta localidad los materiales se obtuvieron de un patio de vivienda en una zona de baja densidad poblacional cuyo proceso de urbanización data de hace 20 años. Este patio posee también plantas exóticas y nativas, ladrillos y troncos que refugian a la fauna estudiada. En ambos casos la colecta de ejemplares fue realizada de manera casual en varias ocasiones a lo largo de 2018; no fueron llevados a cabos censos poblacionales ni conteos exhaustivos de individuos. Los ejemplares colectados han sido fijados en alcohol 70 y depositados en las colecciones de Invertebrados (CFA-In) y artrópodos (CFA-Ar) de la Fundación de Historia Natural «Félix de Azara», Ciudad Autónoma de Buenos Aires, Argentina y en la colección de Aracnología y Miriapodología de la División Aracnología y Miriapodología del Museo de La Plata, Buenos Aires, Argentina (MLP-Ar).

Para evaluar el potencial como invasor de cada especie se realizó una búsqueda bibliográfica y se comentan los perjuicios causados por ellas en base a lo registrado en otros países.

\author{
RESULTADOS \\ Platyhelminthes Claus, 1887 \\ Tricladida Lang, 1884 \\ Geoplanoidea Stimpson, 1857 \\ Bipaliidae Stimpson, 1857 \\ Bipalium kewense Moseley, 1878
}

Material referido.- CFA-In-5561, ejemplar adulto colectado, conservado en alcohol (Figura 1C). El espécimen fue hallado debajo de ladrillos en un jardín interno en el barrio de Liniers, Ciudad Autónoma de Buenos Aires, Argentina. En la misma localidad, distintos ejemplares de esta especie fueron observados en numerosas ocasiones.

Comentarios. - El ejemplar colectado es referible a Bipalium kewense sobre la base de la siguiente combinación de caracteres: extremo anterior de la placa cefálica expandido transversalmente en forma de medialuna y de color grisáceo, con márgenes de color amarillo pálido, cuerpo con color de fondo amarillento, con cinco líneas negruzcas longitudinales, de las cuales la línea media es la mejor definida, línea 


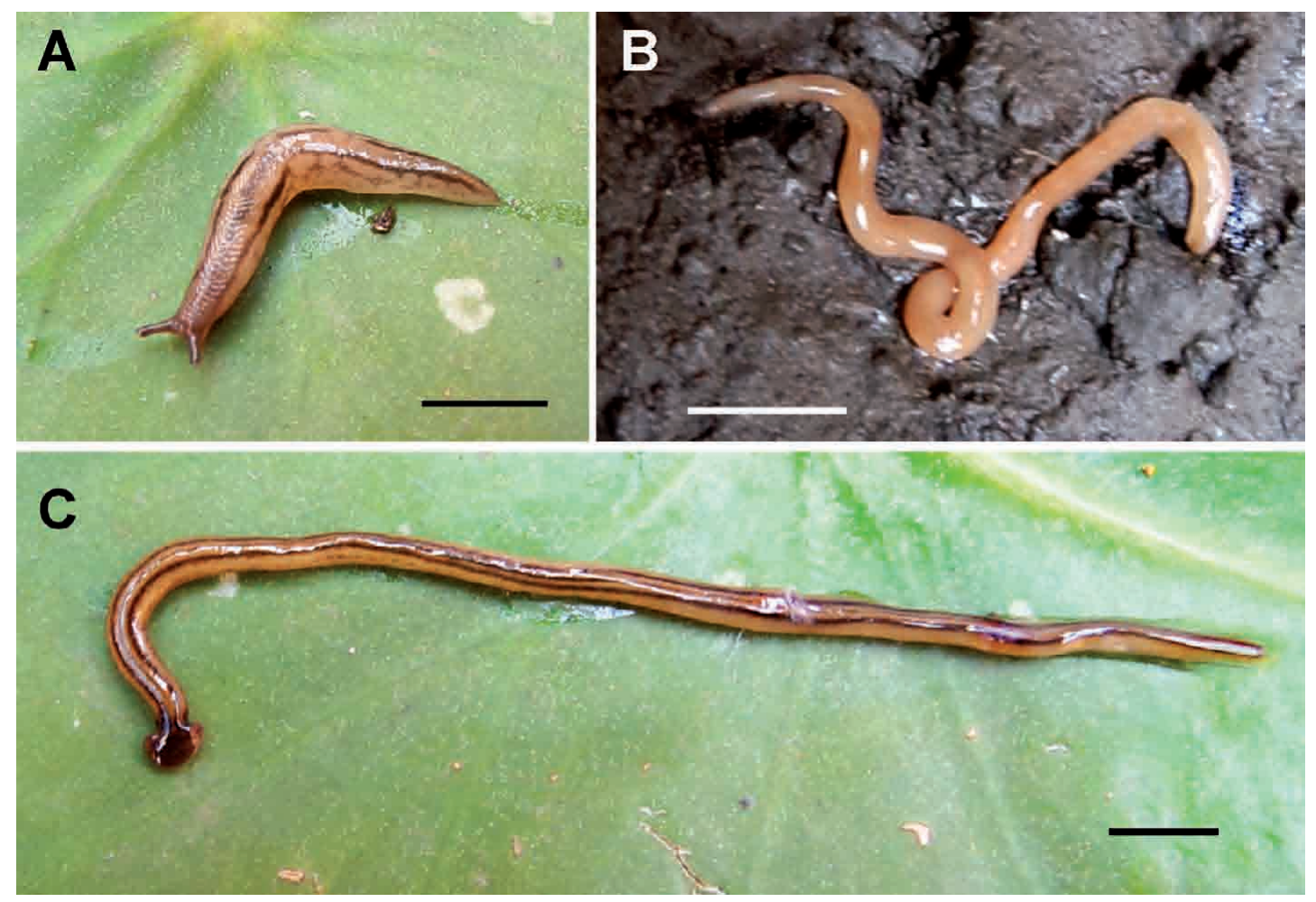

Figura 1. A) Ambigolimax valentianus; B) Austroplana sanguinea; C) Bipalium kewense. Escala = $5 \mathrm{~mm}$.

media no sobrepasa el nivel de la placa cefálica y las líneas marginales forman un cuello discontinuo dorsalmente (Ball, 1977; Winsor, 1983; Sánchez García, 2014). Son de gran tamaño, usualmente alcanzan los 30 o 40 centímetros de longitud (Justine, Thevenoty y Winsor, 2014).

La especie fue originalmente descripta para Inglaterra, aunque su región de origen se radicaría en Asia, posiblemente Indochina (Beauchamp, 1961; Winsor, 1983). Se trata de una especie invasora, ampliamente distribuida en todos los continentes a excepción de Antártida (Winsor, 1983; Morffe, García, Adams y Hasegawa, 2016). Winsor (1983) indica que esta especie en países templados es casi exclusiva de jardines internos, mientras que en regiones subtropicales o tropicales es frecuente en ambientes naturales. Más aún, este autor remarca que los primeros registros de esta especie invasora fueron realizados en ciudades costeras, o cercanas al mar; más recientemente la especie ha sido registrada en ciudades alejadas de la costa, lo que sugiere que la especie se encuentra en constante avance y adaptación en numerosas localidades de Europa, Norteamérica y Asia. Ecológicamente la especie parece estar asociada de manera íntima a los ambientes modificados por el hombre, incluyendo jardines, granjas y tierras cultivadas (Winsor, 1983). Sus posibles presas incluyen larvas de insectos, colémbolos, isópodos, diplópodos, caracoles y lombrices (ver referencias en Winsor, Johns y Barker, 2004).

En Argentina la especie ha sido registrada en las provincias de Tucumán (du Bois-Reymond Marcus, 1953) y Misiones (Negrete y Brusa, 2009). El presente registro constituye el más austral para la especie en el continente y el primero para la provincia de Buenos Aires. 
Geoplanidae Stimpson, 1857

Austroplana sanguinea (Dendy, 1891)

Material referido.- CFA-In-5562, ejemplar adulto colectado, conservado en alcohol (Figura 1B). El espécimen fue hallado debajo de ladrillos en un jardín interno en el barrio de Liniers, Ciudad Autónoma de Buenos Aires, Argentina. En la misma localidad, distintos ejemplares de esta especie fueron observados en numerosas ocasiones.

Comentarios. - El ejemplar colectado presenta una combinación de caracteres externos única que permite referirlo a Austroplana sanguinea: se trata de una especie de tamaño mediano (longitud máxima $80 \mathrm{~mm}$., ancho máximo $5 \mathrm{~mm}$.), transversalmente ancha y aplanada, con el cuerpo en forma de cinta, algo convexo en la línea media y el extremo anterior del cuerpo agudo, coloración característica, rosada, especialmente en su extremo anterior y con leve tinte anaranjado, vientre también rosado, aunque más pálido, presencia de innumerables ojos diminutos en línea desde el extremo anterior y extendidos posteriormente, especialmente concentrados en la región cefálica (Jones, 1981; Mather y Christensen, 1996; Jones, 2005; Justine et al., 2018; Wade, Ames y McKeee, 2018).

Se trata de una especie originaria de Nueva Zelanda (Winsor, 1991), ampliamente distribuida en Australia y Europa, aunque su distribución aún dista de ser clara (Jones, 1981; Mather y Christensen 1996; Winsor et al., 2004; Justine et al., 2014). Al igual que con otras planarias terrestres, se habría dispersado por medio de migración pasiva junto con material vegetal importado (Jones, 1981). Su dieta parece restringirse mayormente a lombrices de tierra (Terrace y Baker, 1994). De hecho, cuando son abundantes pueden incidir negativamente sobre las poblaciones de lombrices (Santoro y Jones, 2001) lo cual podría afectar la agricultura regional a largo plazo (véase Álvarez-Presas, Mateos, Tudó, Jones y Riutort, 2014).

El presente hallazgo constituye el primero para la especie en Sudamérica.

Mollusca Linnaeus, 1758

Limacidae Lamarck, 1801

Ambigolimax valentianus (Férussac, 1823)

Material referido.- CFA-In-5559, ejemplar adulto colectado, conservado en alcohol (Figura 1A). El espécimen fue hallado debajo de ladrillos en un jardín interno en el barrio de Liniers, Ciudad Autónoma de Buenos Aires, Argentina. En la misma localidad, distintos ejemplares de esta especie fueron observados en numerosas ocasiones.

Comentarios.- La especie $A$. valentianus se reconoce claramente sobre la base de una combinación única de caracteres externos: tamaño mediano (longitud máxima de $7,5 \mathrm{~cm}$ ), escudo con margen posterior terminado en punta, quilla longitudinal poco definida en el extremo posterior del cuerpo, coloración de fondo amarillento 
parduzco, con bandas longitudinales negras estrecha, entre 2-3 en el escudo y 2 hasta el borde posterior del cuerpo (Wiktor, De-Niu y W. Ming, 2000; Virgillito, 2012).

Se trata de una especie originaria de la Península Ibérica (Castillejo y Garrido, 1996), invasora en el resto de Europa, Oceanía, África del Sur, Norteamérica, y en Sudamérica citada para Colombia, Perú, Brasil y Chile (Barker, 1999; Agudo-Padrón, 2015). En Argentina se la ha registrado por primera vez en el año 2012 para las provincias de Tucumán, Río Negro, Neuquén y Buenos Aires (Virgillito, 2012). En esta última provincia se la ha reportado para las localidades de Sierras Bayas (Virgillito, 2012) y La Plata (Gutiérrez Gregoric et al., 2013). A pesar de su tardía determinación los ejemplares procedentes de Buenos Aires (Sierras Bayas) datan del año 1924, lo que indica que esta especie se encuentra en nuestro país hace casi 100 años. En la ciudad de Buenos Aires ha sido observado personalmente en gran cantidad de ocasiones y resultó ser el molusco sin caparazón más frecuente luego de Limacus flavus (véase Virgillito y Miquel, 2013).

$A$. valentianus es invasora en jardines en numerosas regiones del globo y suele considerársela un organismo plaga, consumiendo especialmente plantas ornamentales cultivadas (Westervel, 1961; Gutiérrez Gregoric et al., 2013). Se la encuentra en ambientes muy húmedos, siempre bien vegetados (Wiktor, 1989). De hecho, se la considera como una especie perjudicial en los Estados Unidos de Norteamérica (Westervelt, 1961).

A pesar de ser una especie invasora sinantrópica en numerosas localidades y ser generalista tanto en ambientes como en dieta, no tolera climas fríos, lo cual posiblemente sea una importante limitación en su distribución (Mc Donnell, Paine y Gormally, 2009).

Arthropoda Latreille, 1829

Diplopoda de Blainville, 1844

Julidae Leach, 1814

Brachyiulus pusillus (Leach, 1814)

Material referido.- CFA-Ar-252, cuatro ejemplares adultos colectados, conservados en alcohol $70^{\circ}$. Fueron hallados debajo de ladrillos y macetas en un jardín interno en el barrio de Liniers, Ciudad Autónoma de Buenos Aires, Argentina. En la misma localidad, distintos ejemplares de esta especie fueron observados en numerosas ocasiones. Todos los ejemplares al ser molestados se enrollan rápidamente. Se encontró asociado a Oxidus gracilis (Paradoxomatidae) y Armadillidium vulgare (Crustacea, Oniscidea).

Material adicional.- MLP-Ar 20480, cinco ejemplares adultos, conservados en alcohol $70^{\circ}$. Fueron hallados debajo de un tronco en un jardín de una vivienda en la localidad de Romero, partido de La Plata, Buenos Aires, Argentina. Se encontraron junto a Obama sp. (Tricladida, Geoplanidae), Acanthopachylus aculeatus (Arachnida, Gonyleptidae), Asthenoctenus borelli (Arachnida, Ctenidae), Oxidus gracilis (Paradoxomatidae) y Armadillidium vulgare (Crustacea, Oniscidea). 
Comentarios.-Brachyiulus pusillus es un diplópodo de tamaño pequeño (menor de 1,5 centímetros de longitud y 1 milímetro de diámetro) y cuerpo estrecho caracterizable sobre la base de la siguiente combinación de características: número bajo de segmentos (entre 30 y 34), cuerpo de color pardo oscuro, negruzco, con dos líneas longitudinales amarillentas separadas por una línea dorsal longitudinal negruzca (Blower, 1985). Los ejemplares aquí registrados coinciden en un todo con esta combinación de caracteres, y consecuentemente son asignados a esta especie.

$B$. pusillus es una especie típicamente sinantrópica, frecuente en jardines, terrenos baldíos y plantaciones (Sæthre, Staverløkk y Hågvar, 2010). La especie se encuentra debajo de piedras, maderas y escombros, incluso es frecuente en paredes viejas (Andersson et al., 2005), por lo que se trata de una especie sinantrópica estricta, pero de amplio espectro ecológico (Korsós, 1992). Aparentemente el movimiento de tierra y traslado de plantas sería su principal medio dispersor (Korsós, 1992). Se trataría de una especie beneficiosa debido a que contribuiría a airear suelos compactos e inundables (Jeekel y Brugge, 2001).

Sería originaria de la región Mediterránea de Europa (Hoffman, 1999). Esta especie se ha introducido en varios países de Europa, en Estados Unidos, Islas Canarias, África y Australia (Hoffman, 1999; Andersson et al., 2005; Akkari, Stoev, Enghoff y Nouira, 2009; Kime y Enghoff, 2017). Recientemente ha sido citada para Chile (Shelley, Morrill y Morrill, 2014; Golovatch, 2014). La especie ha sido previamente citada como procedente de "Argentina» (Blower, 1985; Hoffman, 1999), aunque sin base material ni especificaciones que respalden la cita.

En la ciudad de Buenos Aires es posiblemente el Diplopoda exótico más común. Se lo encuentra bajo casi cualquier piedra y maceta en todos los jardines urbanos revisados (observación personal).

El presente registro constituye el primero con base material de referencia y localidad precisa en el país. Consecuentemente constituye el primer registro para la especie en la provincia de Buenos Aires.

\section{Paradoxomatidae Daday, 1889 \\ Oxidus gracilis (Koch, 1847)}

Material referido.- CFA-Ar-251, dos ejemplares adultos colectados, conservados en alcohol. Fueron hallados debajo de ladrillos y macetas en un jardín interno en el barrio de Liniers, Ciudad Autónoma de Buenos Aires, Argentina. En la misma localidad, distintos ejemplares de esta especie fueron observados en numerosas ocasiones. Todos los ejemplares al ser molestados se enrollan rápidamente. Se encontró asociado a Brachyiulus pusillus (Julidae) y Armadillidium vulgare (Crustacea, Oniscidea).

Material adicional.- MLP-Ar 20481, dos ejemplares adultos, conservados en alcohol $70^{\circ}$. Fueron hallados debajo de un tronco en un jardín de una vivienda en la localidad de Romero, partido de La Plata, Buenos Aires, Argentina. Se encontraron junto a Obama sp. (Tricladida, Geoplanidae), Acanthopachylus aculeatus (Arachnida, 
Gonyleptidae), Asthenoctenus borelli (Arachnida, Ctenidae), Brachyiulus pusillus (Julidae) y Armadillidium vulgare (Crustacea, Oniscidea).

Comentarios.-Oxidus gracilis presenta una combinación única de caracteres externos que permite su reconocimiento: tamaño usualmente mayor a $15 \mathrm{~mm}$ y menor a $25 \mathrm{~mm}$, cuerpo con entre 18 y 19 segmentos, notorio surco transversal dorsal en cada segmento, coloración de fondo pardo rojiza a negruzca, carenas dorsolaterales prominentes, de color amarillo y bien separadas de los segmentos por una muesca posterior, nunca superan el margen posterior de cada segmento (Blower, 1985; Shelley y Bauer, 1997). Los machos son marcadamente más oscuros que las hembras (Nguyen, Korsós, Jang y Hwang, 2017).

Aunque se desconoce a ciencia cierta, es posible que su distribución nativa sea Japón (Stoev et al., 2010). Se la ha registrado ampliamente en Estados Unidos, Europa, Asia, Australia, y Centroamérica (Hoffman, 1999; Suriel, 2012; Jovanoviæ, Antiæ y Tomix, 2016). Aparentemente el movimiento de tierra y el comercio de plantas sería su principal medio dispersor (Hoffman, 1999).

Es especialmente frecuente en regiones cálidas o templadas, asociada usualmente a ambientes disturbados (Nguyen y Sierwald, 2013). Es una especie con alto potencial adaptativo, lo que le permite poblar varios tipos de ambientes, en especial áreas modificadas por el hombre (Stoev y Korsós, 2010), principalmente jardines internos y viveros (Nguyen y Sierwald, 2013). La especie no es capaz de sobrevivir en ambientes fríos, con temperaturas menores a $-4^{\circ} \mathrm{C}$ (Stoev y Korsós, 2010). Se trata de una especie que es ocasionalmente tratada como plaga, debido a que en ocasiones su densidad puede superar los 2500 individuos por metro cuadrado (Stoev et al., 2010). Se alimenta de vegetales, incluyendo papas, pepinos, raíces diversas y flores cultivadas (Stoev et al., 2010).

La especie ha sido previamente citada como «introducida» en Argentina (Mauriès, 1998), aunque sin base material ni especificaciones que respalden la cita. El presente registro constituye el primero con base material de referencia y localidad precisa en el país. Consecuentemente constituye el primer registro para la especie en la provincia de Buenos Aires.

En la ciudad de Buenos Aires es el segundo Diplopoda invasor más común, luego de Brachyiulus pusillus. Se lo encuentra bajo casi cualquier piedra y maceta en todos los jardines urbanos revisados (observación personal).

Symphyla Ryder, 1880

Scutigerellidae Bagnall 1913

Scutigerella immaculata (Newport, 1845)

Material referido-C CFA-Ar-253, siete ejemplares adultos colectados, conservados en alcohol. Fueron hallados semienterrados entre la tierra, debajo de ladrillos y macetas en un jardín interno en el barrio de Liniers, Ciudad Autónoma de Buenos Aires, Argentina. En la misma localidad, distintos ejemplares de esta especie fueron 
observados en numerosas ocasiones. Se los encontró asociado a Brachyiulus pusillus (Julidae) y Armadillidium vulgare (Crustacea, Oniscidea).

Material adicional.- MLP-Ar 20482, tres ejemplares adultos, conservados en alcohol $70^{\circ}$. Fueron hallados debajo de un ladrillo en un jardín de una vivienda en la localidad de Romero, partido de La Plata, Buenos Aires, Argentina. Se encontraron junto a Armadillidium vulgare (Crustacea, Oniscidea) entre la tierra suelta.

Comentarios. - La especie Scutigerella immaculata puede ser reconocida sobre la base de una combinación única de caracteres visibles con gran aumento: primer par de patas mayor que la mitad del segundo par, cuerpo proporcionalmente robusto, último tergito con una profunda concavidad en su margen posterior, setas anterolaterales dirigidas hacia atrás y fémur del primer par de patas sin espinas (Hansen, 1903; Filinger, 1931; Edwards, 1959; Scheller y Adis, 1996; Scheller, 1998). Dentro de los Symphyla, $S$. immaculata es una especie de gran tamaño, frecuentemente alcanzando los 7-8 milímetros de longitud total (Domínguez Rodríguez, 1992). A simple vista los individuos se muestran como de color blanquecino, diminutos, de antenas largas, con cercos en el extremo posterior del cuerpo, con la apariencia superficial de un ciempiés. Al igual que otros miembros de la familia es de movimientos muy rápidos (Scheller, 1998).

Se trata de una especie que frecuenta ambientes sinantrópicos, de hecho, su nombre común en el hemisferio norte es el de «Garden Symphylid» o Sínfilo de jardín (Filinger, 1931). Es frecuente en pequeños huecos y grietas de la tierra en los jardines, incluyendo espacios dejados por raíces y galerías de lombrices de tierra (Filinger, 1931).

Al igual que otros artrópodos terrestres de escasa movilidad, su gran distribución se debe probablemente a su transporte accidental entre la tierra de plantas comerciables (Filinger, 1931). Es una especie capaz de soportar gran amplitud térmica (entre 9 y $77^{\circ} \mathrm{C}$ ) y gama de precipitaciones (Michelbacher, 1938), aunque los ambientes húmedos parecen ser beneficiosos para su proliferación (Waterhouse, 1968).

S. immaculata es una especie que consume una gran variedad de vegetales (e.g., tomates, pepinos, piñas, lechuga, y numerosos tipos de plantas ornamentales; Filinger, 1931; Agredo, Chaparro y Zuluaga, 1988; Abarca, 1996), especialmente recién germinadas y raíces; cuando es muy abundante puede considerárselo como una plaga (Filinger, 1931; Halliday, 2004).

Se desconoce su localidad geográfica nativa, aunque se encuentra ampliamente distribuida en Europa, Norteamérica, Asia, Australia, África y Sudamérica (Hansen, 1903; Filinger, 1931; Michelbacher, 1938; Savos, 1958; Halliday, 2004; Agredo et al., 1988).

La especie fue citada por primera vez en Argentina para Patagonia (Attems, 1897), aunque este primer registro ha sido desechado por Scheller (1998). Posteriormente, Hansen (1903) registra un lote de 15 ejemplares colectados por F. Silvestri como procedente de «Buenos Aires», sin mayor detalle de localidad. Desde entonces la especie no cuenta con nuevos registros en Argentina (Scheller, 1998).

En la ciudad de Buenos Aires es una especie increíblemente común, que se pue- 
de encontrar ante cualquier palada de tierra o debajo de cualquier objeto que le sirva como refugio. Hemos comprobado su presencia tanto en los dos jardines internos analizados como en otros que visitamos, sin importar que tan pequeños sean; incluso se lo encuentra dentro de macetas aisladas. Los presentes registros constituyen los primeros con base en material de referencia y localidad precisa en el país.

\section{DISCUSIÓN}

Una indeseable consecuencia de la globalización ha sido el notable incremento en el número de especies invasoras que constituyen un reto para la conservación de la biodiversidad y los recursos naturales de distintos ecosistemas (Vitousek, D'antonio, Loope, Rejmanek y Westbrooks, 1997; Hulme, 2009; Simberloff y Vitule, 2014). Las especies invasoras exóticas impactan de manera adversa sobre la biodiversidad, incluyendo la eliminación de especies nativas (a través de competencia por recursos e incluso por transmisión de enfermedades) y la transformación consecuente de ecosistemas locales (Clavero y García-Berthou 2005). Es por eso que su estudio y registro es de especial interés para la conservación de los ambientes. En muchos casos no son las especies exóticas las que producen grandes alteraciones ecológicas, sino que «aprovechan» rápidamente las transformaciones producidas por el ser humano (Didham, Tylianakis, Hutchison, Ewers y Gemmell, 2005). Las especies mencionadas en este trabajo se han establecido en un ambiente urbano con poco o nada de similitud con los ambientes naturales de la región. En este caso, probablemente, lo que les brinda a estas especies exóticas su potencial carácter de «invasivas», es su capacidad de aprovechar la degradación que el ser humano produjo sobre los ambientes naturales. Hasta el momento, en las ciudades estudiadas, las especies tratadas causan daños a los horticultores, pero probablemente no a los ecosistemas nativos que ya se encontraban degradados antes de su introducción.

La dispersión antropocórica pasiva de invertebrados poco vágiles, como ser miriápodos, planarias y moluscos terrestres, parece ser frecuente y ha resultado en invasiones biológicas en todo el mundo (Winsor et al., 2004; Virgillito, 2012; Stoev et al., 2010). En estos casos, los principales responsables parecen haber sido los horticultores, que desde el siglo XIX comercian y transportan plantas exóticas a lo largo de los jardines de todo el mundo, llevando junto con la tierra pequeños invertebrados crípticos (Winsor et al., 2004). Los invertebrados que forman el cuerpo de la presente contribución pueden incluirse en este caso.

Unas 30 especies de Tricladida terrestres se han establecido en países por fuera de su rango natural (Winsor et al., 2004). Estas planarias, incluyendo a Bipalium kewense y Austroplana sanguinea son carnívoras y se alimentan de una gran variedad de organismos del suelo, especialmente lombrices, babosas y caracoles, por lo cual constituyen un riesgo para la biodiversidad local (Alford, Lole y Emmett, 1996; Santoro y Jones, 2001; Jones, Santoro, Boag y Neilson, 2001; Winsor et al., 2004). También producen un impacto negativo sobre la agricultura debido al excesivo consumo de lombrices de tierra (Murchie y Gordon, 2013). Es recomendable el estudio de los impactos de estas especies en la fauna nativa del suelo de Buenos Aires. 
En Argentina los registros de planarias terrestres invasoras son escasos, a pesar de ser formas relativamente abundantes en las colectas y relevamientos faunísticos. Una situación similar ha sido registrada en otros países, siendo considerada por algunos investigadores un vacío en el conocimiento de las faunas locales (Justine, Winsor, Gey, Gros y Thévenot, 2018). Se cuenta con los registros de Caenoplana coerulea para Buenos Aires y Bipalium kewense para Tucumán y Misiones (Meyer y Weyrauch, 1966; Negrete y Brusa, 2009; Negrete, Brussa y Winsor, 2011). Hasta la actualidad, Austroplana sanguinea carece de registros para el país.

El registro más antiguo corresponde a Bipalium kewense en Tucumán, colectado por P. Wygodzinsky y registrado en 1953 (du Bois-Reymond Marcus, 1953). Desde entonces la especie ha sido registrada en Misiones (Negrete y Brusa, 2009) y en Ciudad de Buenos Aires (presente contribución). El clima templado y húmedo de Buenos Aires provee condiciones semejantes a las que estas especies frecuentan en su área original de distribución (Winsor et al., 2004); en consecuencia, no es aventurado proponer que expandirán su distribución geográfica en Argentina, especialmente hacia áreas de clima subtropical o templado. Lamentablemente, aún se carece de un estimativo de cuándo ingresó en nuestro país Austroplana sanguinea.

A diferencia de lo que ocurre con las planarias, el conocimiento de los moluscos invasores ha recibido atención en los últimos años, y existe un cúmulo de información importante sobre las especies introducidas (véase por ejemplo Virgillito, 2012; Virgillito y Miquel, 2013; Gutiérrez Gregoric et al., 2013). En el caso de la Babosa de tres bandas (Ambigolimax valentianus), hasta el año 2012 no se contaba con su presencia en Argentina (Virgillito, 2012). Es probable que los hallazgos cercanos de las localidades de La Plata y Buenos Aires correspondan a una misma población que se podría encontrar en expansión. Esto se sustenta sobre la base de la abundancia de individuos de esta especie en ambas localidades, donde su hallazgo es tan frecuente que sugiere que sus poblaciones cuentan con una excelente salud; adicionalmente ambas ciudades se encuentran conectadas por áreas urbanizadas. En este sentido, Daglio et al. (2016) han indicado que una de las vías de dispersión más comunes para estos moluscos (tanto nativos como exóticos) es el comercio de plantas y tierra, ya que en ellas es probable el traslado de huevos y de ejemplares de poca talla que pasan desapercibidos. Si bien el presente hallazgo no constituye una zona alejada de otras localidades conocidas, representa una cita importante, que posiblemente sea indicativa de la expansión geográfica de esta especie invasora.

En el caso de los miriápodos, el desconocimiento acerca de la distribución e incluso presencia en el país de especies invasoras es casi total. En este respecto, las últimas revisiones regionales sobre sínfilos y diplópodos (Mauriés, 1998; Scheller, 1998) coinciden en señalar que estos grupos en Argentina aún se encuentran muy poco abordados por especialistas. Concretamente, en relación a las especies invasoras, aún poco se conoce. Se citan unas 9 especies introducidas de diplópodos (Mauriés, 1998) de las cuales solo algunas cuentan con material concreto de referencia y localidad precisa (Schubart, 1954a; 1954b; 1954c). De hecho, las especies aquí registradas Oxidus gracilis y Brachyiulus pusillus son por primera vez mencionadas con una localidad precisa, siendo ambas previamente citadas como procedentes de «Argentina» en catálogos generales sin datos que corroboren o indiquen material de 
referencia o alguna localidad (Blower, 1985; Mauriés, 1998). De manera semejante, el sínfilo Scutigerella immaculata, contaba solo con el dato de «Buenos Aires» como localidad, aunque en este caso los ejemplares de referencia fueron brevemente descriptos y adecuadamente conservados, por lo que se tiene alguna certeza acerca de su determinación (Scheller, 1998).

Debido a la falta de otras localidades precisas, se desconoce si las tres especies mencionadas de miriápodos se encuentran en expansión geográfica o demográfica. Sin embargo, debido a la gran abundancia de individuos colectados (las tres especies son muy frecuentes en cualquier plaza, parque o jardín urbano) es posible inferir que sus poblaciones se encuentran bien establecidas y posiblemente en expansión. Vale la pena remarcar que las especies Oxidus gracilis y Scutigerella immaculata pueden encontrarse en grandes números cuando encuentran ambientes favorables, y suelen considerarse plagas debido a los daños importantes que ocasionan en los vegetales cultivados (Filinger, 1931; Stoev et al., 2010).

\section{CONCLUSIONES}

En la presente contribución se realizan citas de invertebrados por primera vez mencionados para la Ciudad Autónoma de Buenos Aires. Estas citas incluyen a los Tricladida Bipalium kewense (Bipaliidae), cuyos registros anteriores se restringían a las provincias de Tucumán y Misiones y Austroplana sanguinea (Geoplanidae), citada por primera vez para Sudamérica y para Argentina. El molusco Ambigolimax valentianus (Limacidae) cuenta con registro en la cercana ciudad de La Plata y en otras regiones del país, por lo que su hallazgo en Ciudad Autónoma de Buenos Aires se puede tomar como un estimativo de la expansión progresiva de su distribución. Los Diplopoda Brachyiulus pusillus (Julidae) y Oxidus gracilis (Paradoxomatidae) cuentan por primera vez con registros concretos para Argentina, mientras que el Symphyla Scutigerella immaculata (Scutigerellidae) poseía solo una cita previa de 1903 que únicamente contaba con el rótulo confuso de «Buenos Aires».

Estos novedosos hallazgos, de especies relativamente comunes y fáciles de encontrar, pero documentadas deficientemente (o aún no documentadas) indican que nuestro conocimiento sobre la fauna de invertebrados invasores es aún muy pobre, y nuevos esfuerzos deben llevarse adelante con la finalidad de catalogar y registrar estas especies introducidas, que pueden impactar de manera adversa sobre la biodiversidad y ambientes naturales y/o causar inconvenientes en las actividades productivas o recreativas. El factor en común en todas las especies citadas en este trabajo es que perjudican en mayor o menor medida a las actividades agropecuarias y horticulturales de diferentes maneras. Una tarea a futuro posiblemente, deba ser la cuantificación de los daños que puedan producir estas especies para establecer su importancia y el costo/beneficio de su control o manejo.

Debido al desconocimiento del registro de todas las especies tratadas en este trabajo, aún ignoramos algunos aspectos básicos de su distribución geográfica. No sabemos, por ejemplo, si algunas de ellas ocupan una gran área geográfica y todos sus hallazgos corresponden a poblaciones interconectadas, o si por el contrario co- 
rresponden a diferentes poblaciones aisladas. Más aún, se desconoce cuando estas especies ingresaron en nuestro país, lo cual imposibilita por ahora estimar tasas de expansión geográfica. Estas consideraciones nos advierten sobre la necesidad del biomonitoreo de estas especies.

\section{AGRADECIMIENTOS}

A las familias Agnolin y Carrión, responsables de los jardines estudiados, por el acceso a ellos. A S. Bogan, quien ha sido de gran ayuda en la determinación de algunos de los ejemplares aquí descriptos. A M. Tassara y L. Pereira por su ayuda en la colección MLP-Ar del Museo de La Plata.

\section{FINANCIAMIENTO}

No se contó con financiamiento para realizar este estudio.

\section{PARTICIPACIÓN}

F.L. Agnolin y A. Agnolin recolectaron los especímenes de Ciudad Autónoma de Buenos Aires y E.L. Guerrero los de La Plata. Todos los autores participaron de la construcción del manuscrito.

\section{LITERATURA CITADA / BIBLIOGRAPHY}

Abarca, G. (1996). Los Sinfilidos (Arthropoda: Symphyla), El complejo de Jobotos (Col: Scarabaeidae) (Phyllophaga spp., Anomala spp. y Cyclocephala spp.) y sus relaciones con la raíz corchosa, en algunos suelos de Costa Rica. En Actas X Congreso Nacional Agronómico, II Congreso de Suelos (63-65). San José de Costa Rica: Costa Rica.

Agredo, C. E., Chaparro, E., Zuluaga, J. I. (1988). Observaciones sobre características, distribución y daños de sinfilidos (Symphyla) y otros organismos del suelo en cultivos de piña, Ananas comosus, del Valle. Acta Agronómica, 38 (2), 65-73.

Agudo-Padron, A. I. (2015). Acerca da ocorrencia de gastropodes exoticos europeios em terras altas do Estado de Santa Catarina, Regiao Central Sul do Brasil. Revista Brasileira de Gestao Ambiental e Sustentabilidade, 2 (3), 203-206.

Akkari, N., Stoev, P., Enghoff, H., Nouira, S. (2009). The millipede order Julida (Myriapoda: Diplopoda) in Tunisia, with an overview of the North African species. Soil organisms, 81 (3), 453-488.

Alford, D. V., Lole, M. J., Emmett, B. J. (1996). Alien terrestrial planarians in England and Wales, and implications for horticultural trade. Proceedings of the Brighton crop protection conference: pests \& diseases, 3, 1083-1088. 
Álvarez-Presas, M., Mateos, E., Tudó, À., Jones, H., Riutort, M. (2014). Diversity of introduced terrestrial flatworms in the Iberian Peninsula: a cautionary tale. PeerJ, 2, e430.

Andersson, G., Meidell, B. A., Scheiller, U., Djursvoll, P., Budd, G., Gardenfors U. (2005). Mångfotingar-Myriapoda. Nationalnyckeln till Sveriges flora och fauna. ArtDatabanken, SLU, Uppsala: Sweden.

Attems, C. M. (1897). Myriopoden. Volume 2, Arthropoden. En Naturhistorisches Museum in Hamburg (1-8), Ergebnisse der Hamburger Magalhaensischen Sammelreise 1892/93. L. Friederichsen \& Co., Hamburgo: Alemania.

Ball, I. R. (1977). La faune terrestre de ĹIle de Saint-Helene, 2. Turbellaria. Annales du Musee Royal de L'Afrique Centrale (Zoologie), 220, 492-511.

Barker, G. M. (1999). Naturalised terrestrial Stylommatophora (Mollusca: Gastropoda). Fauna of New Zealand, 38, 1-247.

Barreiro, G. 2011. Árboles de la ciudad de Buenos Aires. Vázquez Mazini, Buenos Aires, Argentina

Beauchamp, P. (1961). Classe des Turbellaires. Tomo 4. En Traite de Zoologie Anatomie Systemátique Biologie (113-123). Ed. Masson, París: Francia.

Beck, K. G., Zimmerman, K., Schardt, J. D., Stone, J., Lukens, R. R., Reichard, S., Randall, J., Cangelosi, A. A., Cooper, D., Thompson, J. P. (2008). Invasive species defined in a policy context: Recommendations from the Federal Invasive Species Advisory Committee. Invasive Plant Science and Management, 1 (4), 414-421.

Blower, J. G. (1985). Millipedes. Synopses of the British Fauna (New Series) 35. Linnean Society: London.

Carpintero, D. L., De Biase, S., Konopko, S. A. (2014). Hemiptera-Heteroptera de la Reserva Ecológica Costanera Sur (Ciudad Autónoma de Buenos Aires), Argentina. Revista del Museo Argentino de Ciencias Naturales, 16 (1), 67-80.

Castillejo, J. Garrido, C. (1996). Las babosas de la familia Limacidae Rafinesque, 1815 (Gastropoda Pulmonata Terrestria nuda) de la Península Ibérica e Islas Baleares. Morfología y distribución. Nova Acta Científica Compostelana (Bioloxía), 6, 131-143.

Clavero, M., García-Berthou, E. (2005). Invasive species are a leading cause of animal extinctions. Trends in Ecology and Evolution, 20 (3), 110.

Daglio, D., Gutiérrez Gregoric, D. E. (2016). Relevamiento de babosas terrestres de importancia fito-sanitaria en viveros del gran La Plata. Segundo Congreso Argentino de Malacología, 2016, Mendoza, R6.

Didham, R. K., Tylianakis, J. M., Hutchison, M. A., Ewers, R. M., Gemmell, N. J. (2005). Are invasive species the drivers of ecological change? Trends in Ecology and Evolution, 20 (9), 470-474.

Dominguez Rodriguez, M. T. (1992). Shymphyla y Pauropoda (Myriapoda) de suelos de España. Tesis de doctorado, Universidad Autónoma de Madrid: España. Disponible en https://eprints.ucm.es/3690/

du Bois-Reymond Marcus, E. (1953). Some South American Triclads. Anais da Academia Brasileira de Ciencias, 25, 65-78. 
Edwards, C. A. (1959). Keys to the genera of the Symphyla. Journal of the Linnean Society of London, Zoology, 44 (296), 164-169.

Faggi, A. M., Cagnoni, M. (1987). Parque Natural Costanera Sur: las comunidades vegetales. Parodiana, 5 (1), 135-159.

Filinger, G. A. (1931). The Garden Symphylid: Scutigerella immaculata Newport. Bulletin 486, Ohio Agricultural Experiment Station, Wooster, Ohio: Estados Unidos.

Filipello, A. M., Lopez de Casenave, L. (1993). Variación estacional de la comunidad de aves acuáticas de la Reserva Costanera Sur. Revista del Museo Argentino de Ciencias Naturales, 4, 1-15.

Fontanarrosa, M. S., Torres, P. L., Michat, M.C. (2004). Comunidades de insectos acuáticos de charcos temporarios y lagunas en la ciudad de Buenos Aires (Argentina). Revista de la Sociedad Entomológica Argentina, 63 (3-4), 55-65.

Golovatch, S. I. (2014). On some new or poorly-known millipedes from Chile and Argentina (Diplopoda). Russian Entomological Journal, 23 (4), 249-281.

Goodenough, A. (2010). Are the ecological impacts of alien species misrepresented? A review of the «native good, alien bad» philosophy. Community Ecology, 11 (1), 13-21.

Guerrero, E. L. (2014). Nuevos registros de Opiliones (Arachnida) en el este de la provincia de Buenos Aires, Argentina. Historia Natural, tercera serie, 4 (1), 75-84.

Gutiérrez Gregoric, D. E., Beltramino, A. A., Vogler, R. E., Cuezzo, M. G., Núñez, V., Gomes, S. R., Miquel, S. E. (2013). First records of four exotic slugs in Argentina. American Malacological Bulletin, 31 (2), 245-256.

Halliday, R. B. (2004). Confirmation of the presence of Scutigerella immaculata (Newport) in Australia (Symphyla: Scutigerellidae). Australian Journal of Entomology, 43 (1), 43-45.

Hansen, H. J. (1903). The genera and species of the order Symphyla. Quarterly Journal of Microscopical Science, 47, 1-101.

Hoffman, R. L. (1999). Checklist of the millipeds of North and Middle America. Virginia Museum of Natural History, Martinsville, Virginia: Estados Unidos.

Hulme, P. E. (2009). Trade, transport and trouble: managing invasive species pathways in an era of globalization. Journal of Applied Ecology, 46 (1), 10-18.

Hurrell J. A., Delucchi, G., Keller, H., Stampella, P., Guerrero, E. L. (2012). Bryophyllum (Crassulaceae): Especies ornamentales naturalizadas en la Argentina. Bonplandia, 21 (2), 169-181.

Jeekel, C. A. W., Brugge, B. J. H. (2001). Millipedes and centipedes collected in the Kleimeer polder, Province N. Holland, The Netherlands. Myriapod memoranda, 3, 72-93.

Jones, H. D., Santoro, G., Boag, B., Neilson, R. (2001). The diversity of earthworms in 200 Scottish fields and the possible effect of New Zealand flatworms ( $A r-$ thurdendyus triangulatus) on earthworm populations. Annals of Applied Biology, $139,75-92$. 
Jones, H. D. (1981). A specimen of the Australian land planarian Geoplana sanguinea (Moseley) var. alba (Dendy) from the Isles of Scilly. Journal of Natural History, $15(5), 837-843$.

Jones, H. D. (2005). Identification. British land flatworms. British wildlife, 16, 189194.

Jovanoviæ, Z. S., Antiæ, D. Ž., Tomiæ, V. T. (2016). First report of the millipede oxidus gracilis (Diplopoda, Polydesmida, Paradoxosomatidae) in Serbia. Kragujevac Journal of Science, 38, 173-176.

Justine, J. L., Thevenoty, J., Winsor, L. (2014). Les sept plathelminthes invasifs introduits en France. Phytoma-La Défense des végétaux, 674, 28-32.

Justine, J. L., Winsor, L., Gey, D., Gros, P., Thévenot, J. (2018). Giant worms chez moi! Hammerhead flatworms (Platyhelminthes, Geoplanidae, Bipalium spp., Diversibipalium spp.) in metropolitan France and overseas French territories. PeerJ, 6, e4672.

Kime, R. D., Enghoff, H. (2017). Atlas of European millipedes 2: Order Julida (Class Diplopoda). European Journal of Taxonomy, 346, 1-299.

Korsós, Z. (1992). Millipedes from anthropogenic habitats in Hungary. Berichte des Naturwissenschaftlich-Medizinischen Vereins in Innsbruck, Supplementum, $10,237-241$.

Landolfi, M. (2014). Árboles del Parque Avellaneda: Ciudad Autónoma de Buenos Aires: Buenos Aires.

López de Casenave, J., Filipello, A. M. (1995). Las aves acuáticas de la Reserva Costanera Sur: cambios estacionales en la composición específica y en la abundancia de poblaciones y gremios. El Hornero, 14 (1),9-14.

Mather, J. G., Christensen, O. M. (1996). The land planarian Australoplana sanguinea var. alba at a horticultural site in New Zealand. Annals of Applied Biology, 129 (1), 171-179.

Mauriés, J. P. (1998). Diplopoda. En Biodiversidad de Artrópodos Argentinos, una perspectiva biotaxonómica. Ediciones Sur, Buenos Aires: Argentina.

Mc Donnell R. J., Paine, T. D., and Gormally. M. J. (2009). Slugs: a guide to the invasive and native fauna of California. University of California publication no. 8336. Oakland, California: USA.

Meyer, T, Weyrauch, W. K. (1966). Guía para dos Excursiones Biológicas en la Provincia de Tucuman. Miscelánea, 23, 1-127.

Michelbacher, A. (1938). The biology of the garden centipede, Scutigerella immaculata. Hilgardia, 11 (3), 55-148.

Morffe, J., García, N., Adams, B. J., Hasegawa, K. (2016). First record of the land planarian Bipalium kewense Moseley, 1878 (Tricladida: Geoplanidae: Bipaliinae) from Cuba. BioInvasions Records, 5 (3), 127-132.

Mulieri, P. R., Torretta, J. P., Schnack, J. A., Mariluis, J. C. (2006). Calliphoridae (Diptera) of the coastline of Buenos Aires, Argentina: species composition, numerical trends, and bait's preferences. Entomological News, 117 (2), 139148. 
Murchie A. K., Gordon, A. W. (2013). The impact of New Zealand flatworms Arthurdendyus triangulatus on earthworm populations in the field. Biological Invasions, 15 (3), 569-586.

Narosky, T., Henschke, C. (2005). Aves de la Ciudad de Buenos Aires. Vázquez Mazzini Editores, Buenos Aires: Argentina.

Negrete, L. H., Brusa, F. (2009). Las planarias terrestres (Platyhelminthes: Tricladida: Terricola) del Bosque Paranaense argentino. I Congreso Nacional de Ecología y Biología de Suelos CONEBIOS 2009, Universidad Nacional de General Sarmiento, Buenos Aires, R54.

Negrete, L. H., Brusa, F., Winsor, L. (2011). La planaria terrestre azul Caenoplana coerulea, un invasor en Argentina. Revista Mexicana de Biodiversidad, 82 (1), 287-291.

Nguyen, A. D., Korsós, Z., Jang, K. H., Hwang, U. W. (2017). A revision and phylogenetic analysis of the millipede genus Oxidus Cook, 1911 (Polydesmida, Paradoxosomatidae). European Journal of Taxonomy, 293, 1-22.

Nguyen, A. D., Sierwald, P. (2013). A worldwide catalog of the family Paradoxosomatidae Daday, 1889 (Diplopoda: Polydesmida). Check List, 9 (6), 1132-1353.

Núñez Bustos, E. (2008). Las especies urbanas de Rhopalocera de la Reserva Ecológica Costanera Sur, Ciudad de Buenos Aires, Argentina (Lepidoptera: Hesperioidea y Papilionoidea). SHILAP Revista de lepidopterología, 36 (144), 435-447.

Núñez Bustos, E. (2010). Mariposas de la Ciudad de Buenos Aires y alrededores. Vázquez Mazzini Editores, Buenos Aires: Argentina.

Pompozzi, G.A., Peralta, L., Simó, M. (2013). The invasive spider Badumna longinqua (L. Koch, 1867) (Araneae: Desidae) in Argentina: new distributional records, with notes on its expansion and establishment. Check List, 9 (3), 218-221.

Rapoport, E. H. (1996). The flora of Buenos Aires: low richness or mass extinction. International Journal of Ecology and Environmental Sciences, 22, 217-242.

Richardson, D. M. Ricciardi, A. (2013). Misleading criticisms of invasion science: a field guide. Diversity and Distributions, 19 (12), 1461-1467.

Sæthre, M. G., Staverløkk, A., Hågvar, E. B. (2010). Stowaways in horticultural plants imported from the Netherlands, Germany and Denmark. Norwegian Journal of Entomology, 57 (1), 25-35.

Sánchez-García, I. (2014). Cuatro planarias terrestres exóticas nuevas para Andalucía. Revista de la Sociedad Gaditana de Historia Natural, 8, 15-20.

Santoro, G., Jones, H. D. (2001). Comparison of the earthworm population of a garden infested with the Australian land flatworm (Australoplana sanguinea alba) with that of a non-infested garden. Pedobiologia, 45 (4), 313-328.

Savos, M. G. (1958). The bionomics of the garden symphylid, Scutigerella immaculata (Newport). Tesis doctoral. Oregon State College, Oregon: Estados Unidos. Inédita. Disponible en https://ir.library.oregonstate.edu/concern/graduate_thesis_or_dissertations/db78tf410

Scheller, U., Adis, J. (1996). A pictorial key for the symphylan families and genera of the Neotropical Region south of Central Mexico (Myriapoda, Symphyla). Studies on Neotropical Fauna and Environment, 31 (1), 57-61. 
Scheller, U. (1998). Symphyla. En Biodiversidad de Artrópodos Argentinos, una perspectiva biotaxonómica (488-490). Ediciones Sur, Buenos Aires: Argentina.

Schubart, O. (1954). Diplópodos Argentinos del Museo de la Ciudad Eva Perón I. Familia Leptodesmidae. Notas del Museo, 17 (150), 113-146.

Schubart, O. (1954). Diplópodos Argentinos del Museo de la Ciudad Eva Perón II. Familia Sphaerotrichopidae. Notas del Museo, 17 (154), 309-327.

Schubart, O. (1954). Diplópodos Argentinos del Museo de la Ciudad Eva Perón III. Fam. Stylodesmidae, IV. Fam. Cryptodesmidae, V. Fam. Polydesmidae. Notas del Museo, 17, 347-358.

Shelley, R. M., Morrill, E. D., Morrill, D. A. (2014). A julid milliped in Chilean Patagonia, and a compilation of ordinal representatives in South America and associated islands (Diplopoda: Julida). Insecta Mundi, 2014, 0363-0366.

Simberloff, D. (2005). Non-native species do threaten the natural environment! Journal of Agricultural and Environmental Ethics, 18 (6), 595-607.

Simberloff, D., Vitule J. R. S. (2014). A call for an end to calls for the end of invasion biology. Oikos, 123, 408-13.

Stoev, P., M. Zapparoli, S. Golovatch, H. Enghoff, N. Akkari, Barber, A. (2010). Myriapods (Myriapoda). Chapter 7.2. In: Roques, A., Kenis, M., Lees, D., Lopez-Vaamonde, C., Rabitsch, W., Rasplus, J.-Y., Roy, B.D. (eds). BioRisk, Special Issue, Alien terrestrial arthropods of Europe, 4 (1), 97-130.

Suriel, C. (2012). Presencia de Oxidus gracilis, Chondromorpha xanthotricha y Asiomorpha coarctata (Diplopoda: Paradoxosomatidae) en La Hispaniola. Solenodon, 10 .

Terrace, T. E., and Baker G. H. (1994). The blue land planarian, Caenoplana coerulea Moseley (Tricladida: Geoplanidae), a predator of Ommatoiulus moreleti (Lucas) (Diplopoda: Julidae) in southern Australia. Australian Journal of Entomology, 33, 371-372.

Virgillito, M. (2012). Panorama de los gastrópodos terrestres exóticos en la Argentina (Gastropoda Pulmonata Stylommatophora). Facultad de Ciencias Exactas y Naturales, Universidad de Buenos Aires, Buenos Aires. Tesis de grado, inédita.

Virgillito, M., Miquel S. E. (2013). New records of exotic land snails and slugs in Argentina. Revista del Museo Argentino de Ciencias Naturales 15 (2), 295-303.

Vitousek, P. M., D’antonio, C. M., Loope, L. L., Rejmanek, M., Westbrooks, R. (1997). Introduced species: a significant component of human-caused global change. New Zealand Journal of Ecology, 21 (1), 1-16.

Wade, M., Ames, V., McKeee, K. (2019). Australian Flatworm. GB Non-native species secretariat. Disponible online en www.nonnativespecies.org/downloadDocument.cfm? $\mathrm{id}=349$

Waterhouse, J. S. (1968). Studies on the garden symphylan, Scutigerella immaculata (Symphyla: Scutigerellidae). The Canadian Entomologist, 100, 172-178.

Westervelt, C. A. (1961). The biology and systematic status of Limax (Lehmannia) valentianus Férussac 1823. Unpublished thesis, University of Arizona, Arizona: Estados Unidos. 
Wiktor, A. (1989). Limacoidea et Zonitoidea nuda. Slimaki pomrowiokszta ${ }^{3}$ tne (Gastropoda: Stylommatophora). Fauna Poloniae 12. Warsaw, Poland: Polska Akademia Nauk.

Wiktor, A., De-Niu, C., Ming, W. (2000). Stylommatophoran slugs of China (Gastropoda: Pulmonata)-Prodromus. Folia Malacologica, 8 (1), 3-35.

Winsor, L., Johns, P. M., Barker, G. M. (2004). Terrestrial planarians (Platyhelminthes: Tricladida: Terricola) predaceous on terrestrial gastropods. En Natural enemies of terrestrial molluscs (227-278). Oxfordshire: CAB International.

Winsor, L. (1983). A revision of the cosmopolitan land planarian Bipalium kewense Moseley, 1878 (Turbellaria: Tricladida: Terricola). Zoological Journal of the Linnean Society, 79 (1), 61-100.

Winsor, L. (1991). A provisional classification of Australian terrestrial geoplanid flatworms (Tricladida: Terricola: Geoplanidae). Victorian Naturalist (Blackburn), 108 (2), 42-49.

Zapata, L. V., Grismado, C. J. (2015). Lista sistemática de arañas (Arachnida: Araneae) de la Reserva Ecológica Costanera Sur (Ciudad Autónoma de Buenos Aires, Argentina), con notas sobre su taxonomía y distribución. Revista del Museo Argentino de Ciencias Naturales, 17 (2), 183-211. 